\title{
Vulture-Based AdaBoost-Feedforward Neural Frame Work for COVID-19 Prediction and Severity Analysis System
}

\author{
S. Roselin Mary ${ }^{1}$ (D) Vinit Kumar $^{2} \cdot$ K. J. Prasanna Venkatesan ${ }^{3} \cdot$ R. Satish Kumar ${ }^{4} \cdot$ Naga Padmaja Jagini $^{5}$. \\ Amedapu Srinivas 6
}

Received: 22 October 2021 / Revised: 20 January 2022 / Accepted: 28 January 2022 / Published online: 22 February 2022

(c) International Association of Scientists in the Interdisciplinary Areas 2022

\begin{abstract}
In today's scenario, many scientists and medical researchers have been involved in deep research for discovering the desired medicine to reduce the spread of COVID-19 disease. However, still, it is not the end. Hence, predicting the COVID possibility in an early stage is the most required matter to reduce the death risks. Therefore, many researchers have focused on designing an early prediction mechanism in the basis of deep learning (DL), machine learning (Ml), etc., on detecting the COVID virus and severity in the human body in an earlier stage. However, the complexity of X-ray images has made it difficult to attain the finest prediction accuracy. Hence, the present research work has aimed to develop a novel Vulture Based Adaboost-Feedforward Neural (VbAFN) scheme to forecast the COVID-19 severity early. Here, the chest X-ray images were employed to identify the COVID risk feature in humans. The preprocessing function is done in the initial phase; the error-free data is imported to the classification layer for the feature extraction and segmentation process. This investigation aims to track and segment the affected parts from the trained X-ray images by the vulture fitness and to segment them with a good exactness rate. Subsequently, the designed model has gained a better segmentation accuracy of $99.9 \%$ and a lower error rate of 0.0145 , which is better than other compared models. Hence, this proposed model in medical applications will offer the finest results.
\end{abstract}

\section{Graphical abstract}

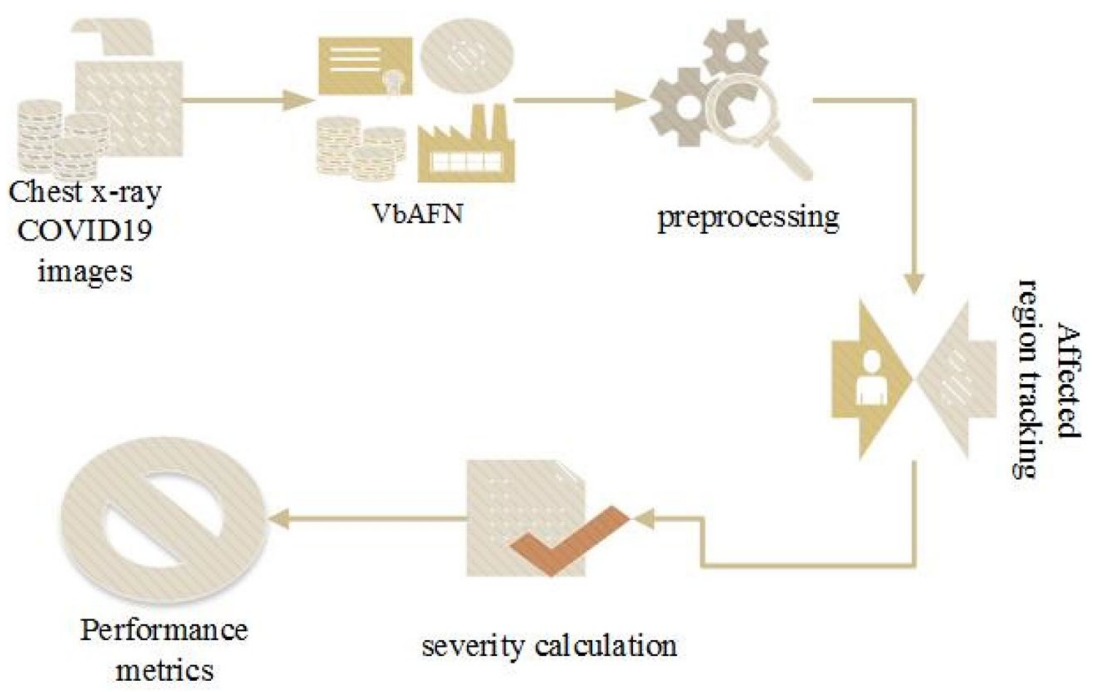

Keywords Affected part tracking $\cdot$ Chest X-ray $\cdot$ COVID-19 $\cdot$ Feature extraction $\cdot$ Segmentation $\cdot$ Severity analysis

Extended author information available on the last page of the article 


\section{Introduction}

One of the infectious diseases is called COVID-19 that is emerged in China in 2019 December [1]. The reason for implementing the early prediction system for COVID disease is, it has affected the respiratory system and made people critical [2]. Besides, this disease is vulnerable for older people, especially those affected by cardiovascular, diabetes, cancer, and respiratory diseases [3]. Therefore, to predict the COVID affection, the medical system has utilized the advances of the imaging system [4]. Considering other systems such as numerical prediction by neural models, digital image processing has provided the finest results in predicting and segmenting the COVID-affected part [5]. In addition, it is one of the most deadly diseases for humans in the present decade are COVID-19; it has affected the human's breathing system [6]. In addition, this COVID pandemic is increasing the challenges day by day in the medical fields [7]. In addition, it has maximized the necessity of hospitals, medical equipment, hospital beds, and so on [8]. Thus, there is a shortage in quick medical health decisions and medical resources. Moreover, an intelligent system such as a neural model is utilized efficiently to predict and segment the COVID-affected part from the X-ray chest images [9].

An effective screening has enabled the rapid and proficient COVID-19 prediction framework, which can reduce the complexity on the healthcare system [10]. In recent, much literature has been played a significant role in studying and analyzing the COVID-19 severity [11]. However, in many cases, it was reported that predicting the COVID disease is one of the most time-consuming tasks because of data set complexity [12]. The basic of the disease prediction model is illustrated in Fig. 1.

However, early detection is an important measure to reduce the death rate and improve human health [13]. Previously, numerous approaches were developed to predict the COVID-19 severity early, such as DL [18, 19], artificial neural model [20], etc., but still, an accurate solution is not found. Therefore, the present research has proposed a novel optimized neural framework to improve prediction accuracy. Moreover, the proposed approach is the hybrid paradigm with the ensemble and deep learning strategies. In addition, to attain the finest tracking and segmentation results, vulture fitness was upgraded in the classification layer of the hybrid neural model. The remaining section is summarized as follows: Sect. 2 investigates recent related works based on the COVID-19 cell detection. Section 3 provides the existing system and its problem definition, and Sect. 4 demonstrates the proposed work process. Besides, investigational outcomes are comprehensived in Sects. 5 and 6 ends the article.

\section{Related Works}

Recent associated works are discussed as follows:

In several worldwide researchers are discovering effective procedures to forecast the severity of COVID-19, But it met several issues, because the prediction of COVID-19 earlier stage is very difficult. Therefore, Shang et al. [26] planned a regression replica to predict the CONID 19 disease and
Fig. 1 Artificial intelligence (AI) technique vs COVID-19

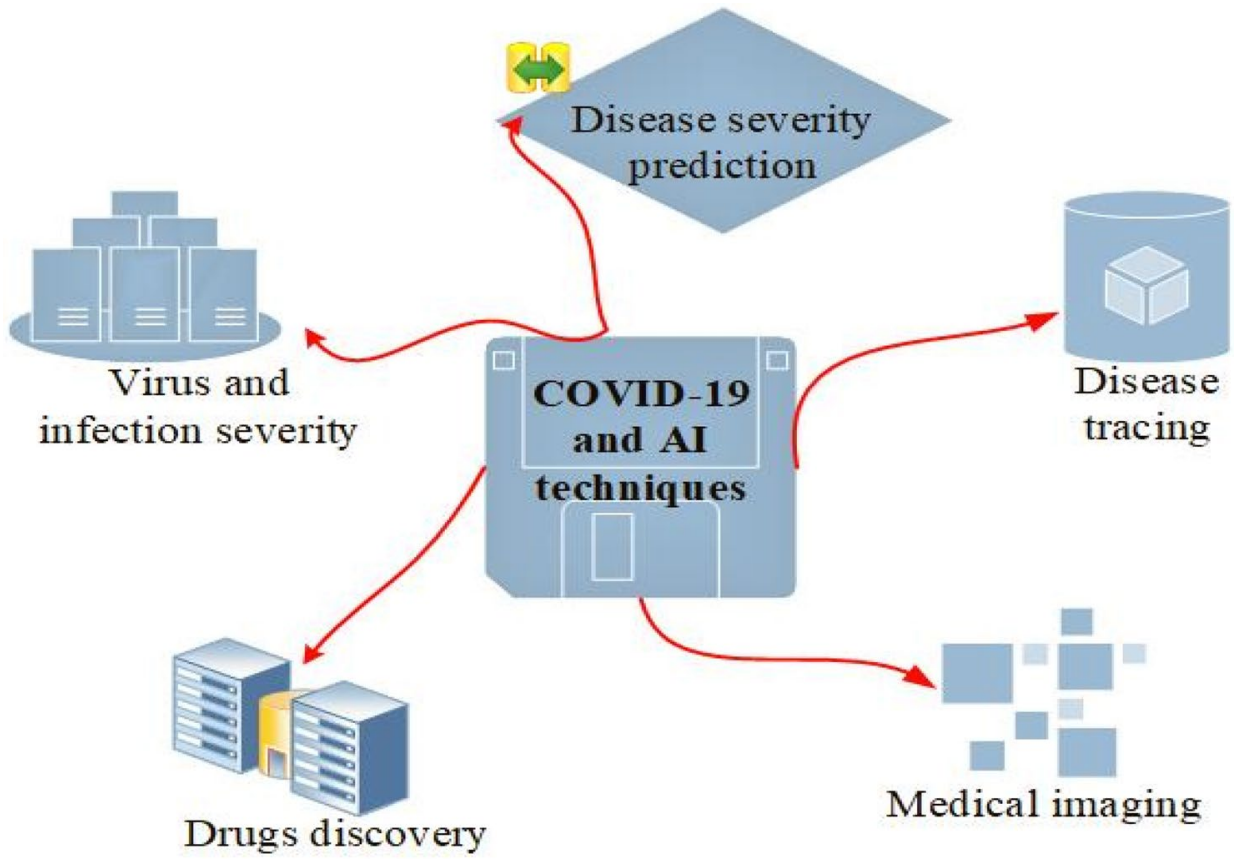


to reduce the training error. Consequently, the effectiveness of the projected model is compared with other existing approaches and has attained good diagnosis accuracy. Nevertheless, it attained high computation time because of reduced training error.

Therefore, Cheema et al. [27] have introduced a novel intelligent computing standard named Levenberg based artificial neural framework to predict the affected peoples early. Moreover, the proposed function is also used as a nonlinear differential equation for effective performance. Here, the training and testing process is done with the help of the back propagation model of the proposed neural framework. However, in some cases of complex data has attained very little accuracy.

Shoaib et al. [28] have introduced a hybrid nonlinear autoregressive-based radial function to improve the prediction accuracy. Furthermore, the proposed transfer model converts the local values into global values after finishing the bimodal implementation. Here, the nonlinear mathematical model achieves higher accuracy, sensitivity, etc. Consequently, the finest outcomes of the proposed model are validated with another paradigm in terms of MSE, RMSE. However, it takes more time to perform the entire process.

Zhang et al. [29] developed the AI-based novel coronavirus pneumonia pattern to improve the prediction performance. The key aim of this work is to predict the acute infection and enhance the treatment performance of COVID-19 disease. When the health condition is overloaded proposed replica is incorporated with segmentation networks to analyze the affection severity. Clinical features and prognosis analysis collapsed during the classification process, so the proposed model could not obtain smoother and clearer boundaries.

The COVID-19 disease has affected millions of human beings and caused more death rates worldwide. Therefore, Shanbhag et al. [30] have designed the ML-based mortality prediction model, which was applied to the clinical data set. Here, 961 data sets are worn for the preparation process, and 249 data sets are used as the testing procedure. Moreover, the extraction process is done on specific rules, and this approach has not reached the accurate level of prediction accuracy. However, it takes more time to process.

The key steps of the research are described as,

- Initially, the Chest X-ray data set is collected from the Kaggle site [31] and trained to the MATLAB system, this data set contains chest X-ray images and CSV files, the total size of the file is $1.29 \mathrm{~GB}$.

- Then the training errors are removed in the preprocessing layer.

- Hereafter the preprocessed image is entered into the classification layer of the optimized ensemble model named VbAFN.
- Then the diseases are classified by segmenting the affected part in the testing phase with the help of trained sets.

- The presence of the optimized fitness value in the designed AdaBoostFeedforward neural system's dense layer will improve the classification and segmentation accuracy.

- Finally, the proposed work is validated by calculating and comparing the key metrics in terms of accuracy, recall sensitivity, error rate, F-measure, and precision.

\section{System Model and Problem Statement}

Usually, image processing techniques are used in the medical field for better disease validation and severity estimation [14]. These days, the affection of COVID-19 differs from each individual differently [15]. Most of the affected people are going to the hospital at a severe stage, so the recovery rate is very low. Moreover, several investigations are done previously to predict the COVID-19 disease symptoms for an early stage in a particular human part and measure that severity [16].

Nowadays, the disease COVID-19 became the most deadly disease globally, so it turns the attention to research severity prediction criteria [17]. In addition, predicting the COVID symptom will help recover quickly and minimize the death rate. However, the prediction of COVID symptoms in the earlier stage is not an easy task by the conventional models, which is mentioned in Fig. 2. In this work, the medical data set is planned to take that is related to COVID19. The data sets such as the Chest X-ray image data set is planned to use in this research.

\section{Proposed VbAFN for COVID-19 Disease Prediction}

The key motive of this present research work is to recognize and classify the COVID-19 from the trained database. Moreover, this research aims to predict the COVID-19 severity at an earlier stage to improve health. Hence, to recognize and segment the COVID-19 cell from the Chest X-ray data set, a novel VbAFN Framework was designed. Moreover, incorporating the vulture fitness function in the Adaboost dense layer can help gain the best severity classification results. The presented architecture is detailed in Fig. 3.

Initially, X-ray data sets are collected from the net source and trained with the help of the proposed framework. Hereafter, the trained data sets are submitted to the preprocessing stage to remove the redundant information. 
Fig. 2 System model with the problem statement

\section{Chest X-ray dataset}

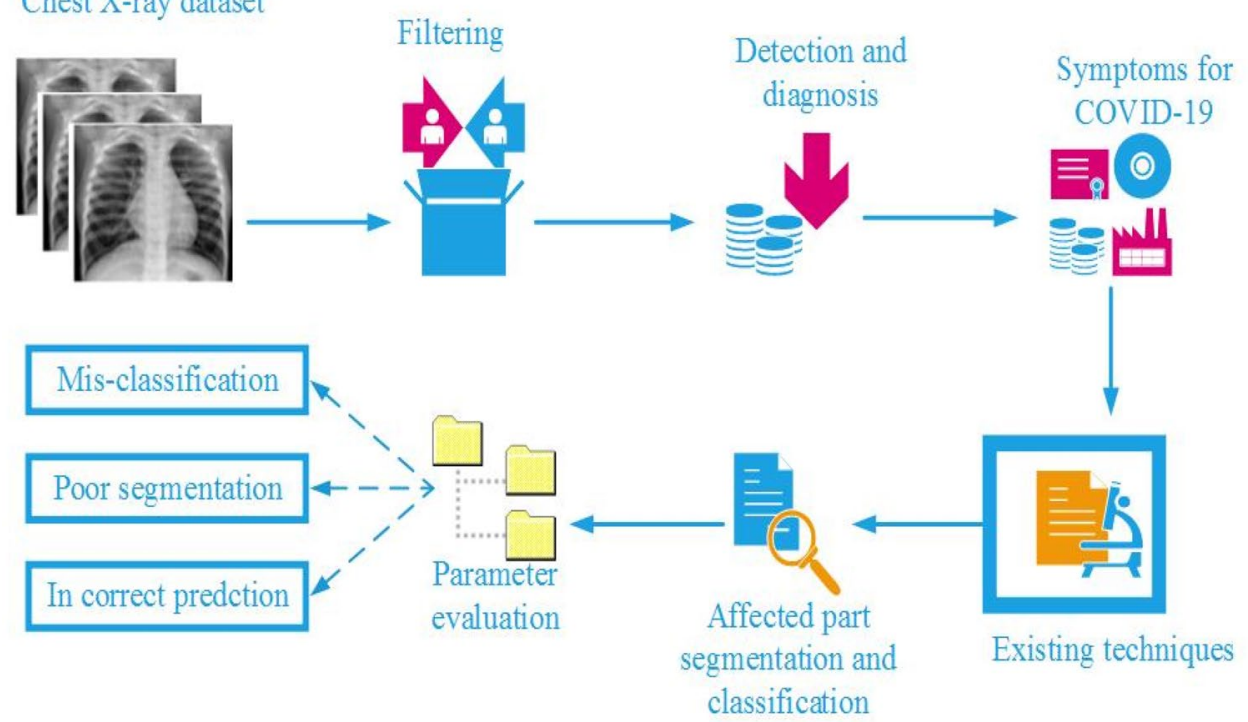

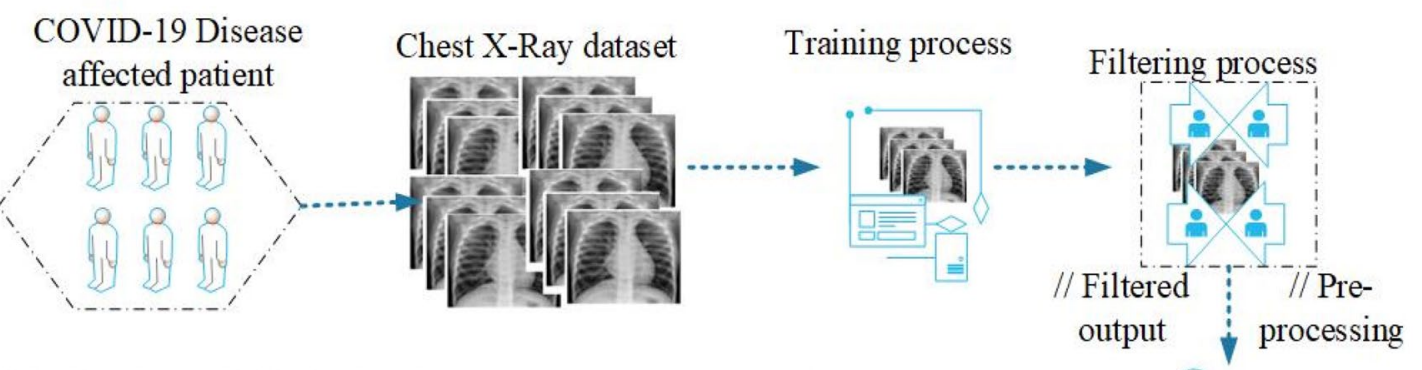

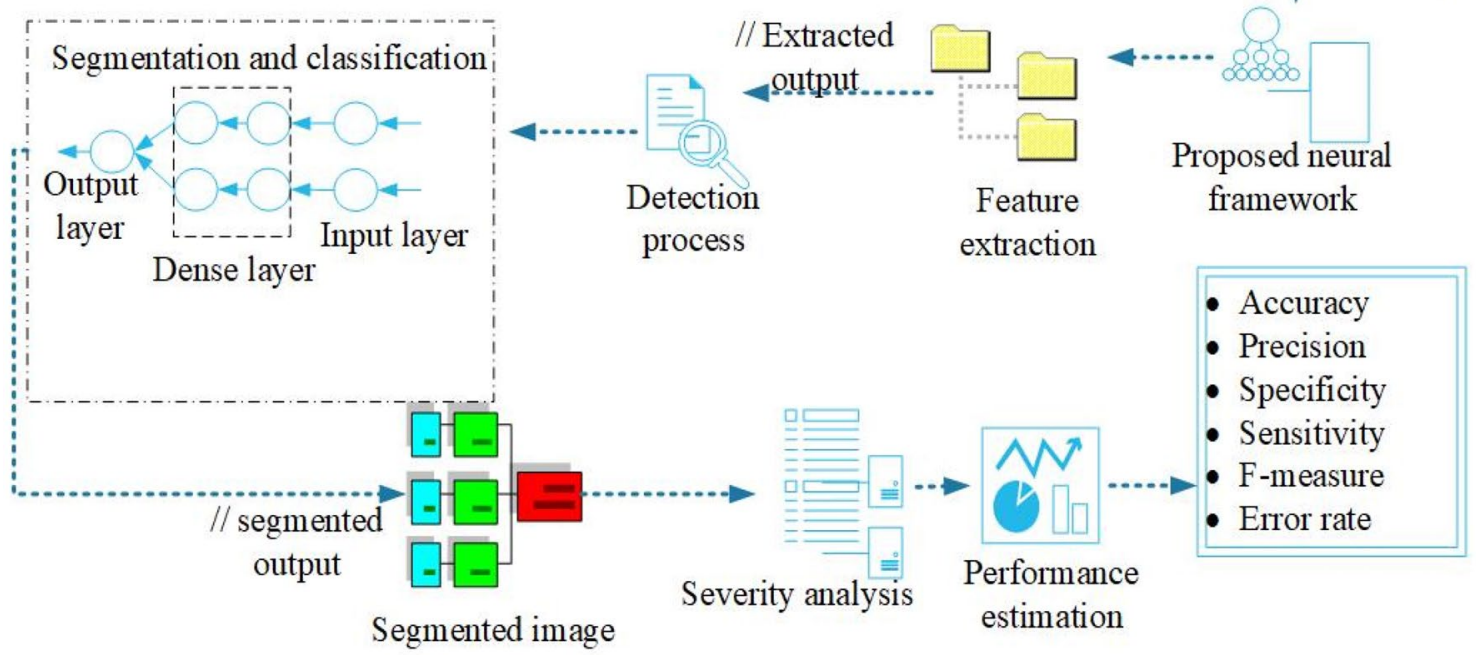

Fig. 3 Proposed VbAFN design

Consequently, the proposed VbAFN model has utilized the X-ray data set to classify and segment the severity of COVID-19 in an earlier stage and enhance the health condition.

\subsection{Data Set Description}

The check X-ray data set is collected from the Kaggle site, chest X-ray images. The total size of the data set is $1.29 \mathrm{~GB}$. 
In that 10,000 images are there, $30 \%$ testing and $70 \%$ images for training were utilized in this research. In addition, there are 810 normal Chest X-ray images and 9190 COVIDaffected images that have included less severity to high severity. In addition, in the trained 7000 images, 500 images are normal images, and 6500 images are abnormal. In addition, in the tested 3000 images, 310 images are normal, and 2690 images are abnormal. Here, the abnormal data sets are considered for feature extraction and classification purposes. Moreover, the method that has been adapted to track and segment the disease-affected region is DL-ensemble learning with an optimization model.

\subsection{Process of VbAFN Model}

In this study, the VbAFN strategy is developed for recognizing and segmenting the severity of COVID-19 cells from the Chest X-ray data set. Here, the chest X-ray data set is taken from the Kaggle website. Moreover, the proposed technique combines vulture optimization and the Adaboost feed-forward neural framework. Here, the fitness function of vulture is updated into the dense layer of the neural framework to enhance the segmentation and classification accuracy. AFN is the dynamic operational scheme, which is incorporated for decreasing image analyzing complexity by reducing the training noise. Moreover, these newly created neurons are attached to the vulture model member function. In a vulture optimization, first, update all the solution sets; here, chest $\mathrm{X}$-ray data sets are considered. Hereafter, the vulture fitness, such as the tossing of pebbles, takes place to track the affected region from the chest X-ray data sets. This fitness function was repeated again and again till the best tracking results. Initially, the collected chest X-ray data sets are collected for various COVID-19-affected patients initiated to the developed system, which is denoted as in the following equation:

$D(s)=\left\{d_{n}^{\prime}, C_{s}\right\} \Rightarrow n=1,2, \ldots \ldots \ldots \ldots . . . k$

where $D(s)$ is the data set representation, $d_{n}^{\prime}$ is represented as collected chest X-ray data sets for COVID-19 disease and $C_{s}$ is denoted as symptoms for COVID-19 disease. Moreover, $n$ denotes the patients, which is mentioned in $n=1,2, \ldots \ldots \ldots \ldots . . . k$. Consequently, training errors are removed using the developed system.

Initially, the Adaboost classifier chose the training data sets randomly. Here, the classifier is incorporated with a feed-forward framework to segment and track the affected part. It schedules the maximum weight to inaccurate classified data sets; therefore, the next iteration, these classified images attain a higher probability for the classification process.

Pre-processing: Chest X-ray images have more unpredictable and redundant information or errors, reducing image identification and image resolution. Therefore, the preprocessing layer is used to improve the image quality to reduce the unwanted information present in the collected data set. Here, the kernel function is used for the preprocessing process. The collected data set input width and height is initiated in the kernel function of the proposed framework, which is mentioned in the following equation:

$R(x)=d_{n}^{\prime}(x) * e^{\prime}(x)=\sum_{t=-\infty}^{+\infty} d_{n}^{\prime}(t) e^{\prime}(x-t)$,

where $d_{n}^{\prime}$ is denoted as input data set that is collected chest $\mathrm{X}$-ray data set, $e$ is the parameter representation of kernel function, $\mathrm{t}$ is the time delay, and $\mathrm{x}$ is the time. Moreover, the kernel function is represented as $d_{n}^{\prime}(a, b)$ and $e^{\prime}(a, b)$, then, the error removed output data sets are denoted as $P(a, b)$ that is mentioned in the following equation:

$P(a, b)=d_{n}^{\prime}(a, b) * e \prime(a, b)$.

The errors and unwanted noises are removed from the collected data set. The preprocessing stage enhances the chest X-ray image portion, which comprises the undesired alterations and expands the several important image features for further processing. Therefore, the kernel function effectively eliminates the undesired noises in the chest X-ray images and the processed images are moved to the process.

Affected region tracking: At first, COVID-19 disease has normally terminated the chest and their connection in the parts of lungs involved in the alveoli. Moreover, the lung is the most affected region of the COVID-19 disease. Affected part tracking contains three steps: feature extraction, segmentation, and classification. Region tracking steps are done with the help of optimization fitness function and neural framework.

Feature extraction: in image processing, feature extraction has termed the process of dimensionality reduction based on the image characteristics. The preprocessed data set is separated and decreased to more manageable data important for detection and categorization. In addition, the process of feature extraction improves the accuracy when extracting the features from the input data set. Here, the nonlinear function is used as a facet extraction process that is mentioned in the following equation:

$F(e)=\frac{d_{n}^{\prime} * g\left(d_{n}^{\prime}\right)}{P_{x}\left(d_{n}^{\prime}\right)}$

where $g\left(d_{n}^{\prime}\right)$ is denoted as grey level values of each image and $P_{x}\left(d_{n}^{\prime}\right)$ is represented as pixel range of each preprocessed data set. Moreover, the extracted features are selected rationally to implement the preferred classification image in that area chosen instead of considering the entire area. Here, the 
features are extracted and moved to the dense layer to categorize the COVID-19 severity analysis.

Segmentation and classification: normally, segmentation is the process of dividing the collected chest X-ray data set into various portions according to its properties, attributes, and features. The image segmentation process is done on pixel accuracy. In a segmentation process, the extracted features from the collected chest X-ray data sets are moved to the dense layer of the AFN framework. In this, the optimized fitness function is updated into the AFN dense layer to segment and detect the affected parts. Therefore, the process of segmentation is calculated using the following equation:
$S\left(d_{n}^{\prime}\right)=\frac{F(e) * P_{x}\left(d_{n}^{\prime}\right)}{V(f)}$

where $S\left(d_{n}^{\prime}\right)$ is referred to as segmented output of the affected part in the chest and $v(f)$ is vulture fitness that is egg breaking. Consequently, the egg breaking fitness is updated in the dense layer to segment and easily classify the affected part. In addition, COVID-19 severity predicts based on the vulture fitness. At last, the output layer provides the segmented and classified output. Here, the affected part is segmented using the proposed VbAFN framework detailed in Algorithm 1.

\begin{tabular}{|c|c|}
\hline & Algorithm 1 Proposed VbAFN framework \\
\hline & Input: chest $X$-ray datasets \\
\hline \multirow[t]{28}{*}{ Start } & \\
\hline & Initialization \\
\hline & \{ \\
\hline & // based on the solution set initialization process \\
\hline & \} \\
\hline & Training process \\
\hline & for $(n=1 ; n \geq k ; n++)$ \\
\hline & Initiate the symptoms \\
\hline & $D(s) \Rightarrow C_{s} \quad / / C_{s}$ is symptoms for COVID 19 disease \\
\hline & End for \\
\hline & Perform the operation of $A F N$ \\
\hline & Pre-processing // using kernel function $\left(e^{\prime}\right)$ \\
\hline & $\forall D(s)$ \\
\hline & $D(s) D(s) \Rightarrow d_{n}^{\prime}\left(e^{\prime}\right)$ \\
\hline & Preprocessed dataset \\
\hline & // Affected region tracking \\
\hline & feature extraction, segmentation and classification \\
\hline & feature extraction \\
\hline & Update preprocessed chest $X$-ray datasets \\
\hline & $\boldsymbol{F o r}(n=1,2, \ldots \ldots \ldots \ldots . . . k)$ \\
\hline & features are extracted \\
\hline & End for \\
\hline & Segmentation and classification \\
\hline & Update the vulture fitness \\
\hline & //Update extracted image \\
\hline & Classify the images using segmented output \\
\hline & Severity analysis \\
\hline & Finest solution \\
\hline \multirow[t]{2}{*}{ Stop } & \\
\hline & Output: segmented image \\
\hline
\end{tabular}




\section{Results and Discussion}

The developed VbAFN technique implementation is done on the MATLAB 2018Rb running on Windows 7 platform. The initial step of the proposed system is training, which is to train the collected data set with all COVID-19. After, that takes some of the symptoms related to the COVID-19 such as trained to the machine to forecast the COVID-19 disease severity.

\subsection{Case Study}

In the modern era, COVID-19 is a dangerous disease that faces human beings in specific. Moreover, in 2020 many people are died because of the COVID-19 disease, which creates higher death percentages worldwide. Previously, different medical images were used to find the severity of this affected disease. However, here, X-ray images are taken, which is more efficient to predict the affected parts and analysis their severity. In this technique, chest X-ray data sets are collected and trained with the help of the MATLAB system. The data set chest X-ray input images for a two patients are taken in Fig. 4.

Consequently, the filtered input images are performed the feature extraction process that are removed the unwanted errors in the images, which are done in the proposed model. After the training process, the trained images are moved to the process to remove the redundant information from the trained images.

Then, apply the fitness function in the dense layer of the proposed neural framework. It will improve the segmentation and classification accuracy of the developed technique. In addition, it recognizes the affected parts separately, which is mentioned in Fig. 5. From that segmented image severity of the COVID-19 is analyzed.

Moreover, $70 \%$ of data has been taken as the training process, and the outstanding data has been taken as the test procedure. Consequently, training accuracy and correspondent loss of the developed VbANF model is demonstrated in Fig. 6.

Fig. 4 Input Chest X-ray: a sample 1 , b sample 2
Moreover, the training accuracy is increased over the validation accuracy with a particular epoch. The training and validation loss is decreased significantly to the overall epochs.

\section{Performance Evaluation}

The proposed VbANF method analyses the severity of COVID-19 disease from chest X-ray images and segments the affected part. Therefore, the proposed replica has attained the finest outcomes in terms of Precision, specificity, sensitivity, f-measure, etc. Moreover, the testing performance of the proposed VbANF method confusion matrix is important. Here, the confusion matrix is the framework for calculating the results based on the classified accurate and inaccurate count values.

In addition, the overall performance of the developed VbANF technique is demonstrated in Fig. 7. Here, the developed VbANF technique has attained 99\% segmentation accuracy and $99 \%$ precision for 10 data sets.

In addition, $98.02 \%$ specificity and $99.1 \%$ sensitivity are obtained for 10 data sets mentioned in Fig. 8. Consequently, the developed model has attained an F1 score of $97.23 \%$ and a $0.014 \%$ error rate for the same 10 data sets.

\subsection{Comparative Analysis}

The planned model is executed in MATLAB, and the successive score was calculated by comparing the key metrics with old models in terms of, accuracy, Precision, sensitivity, error rate, F-measure, and recall. CNN with Fuzzy (CNN-F) [21], Fusion schemes (FC) [22], bat optimization with Fuzzy (BO-F) technique [23], CNN with VGG16 [24], and Hidden Markov with U-net Architecture (HMUA) [25].

\subsubsection{Accuracy}

Accuracy is the ratio that accurately predicts the severity of COVID-19 performance to the entire performance.

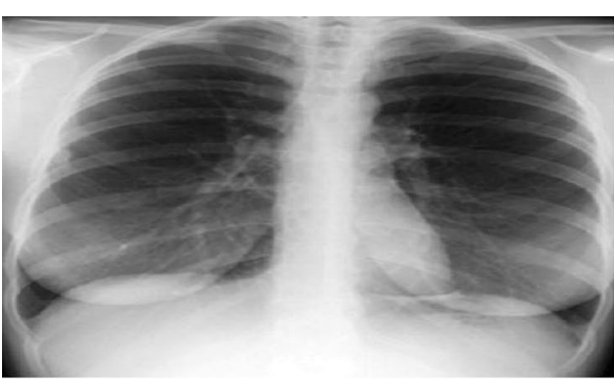

(a)

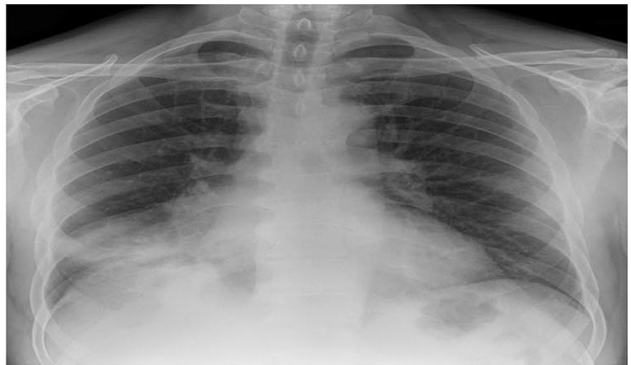

(b) 


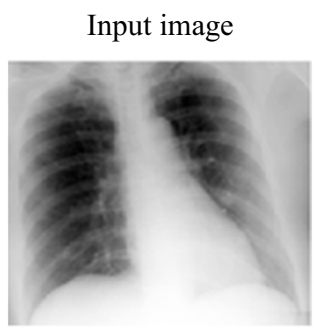

(a)

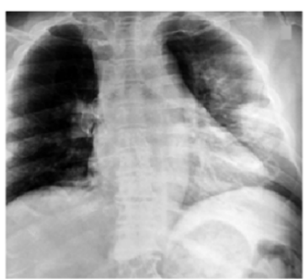

(e)

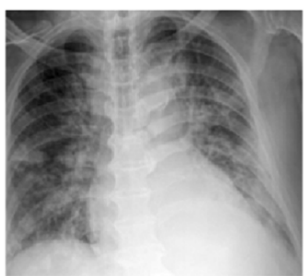

(i)

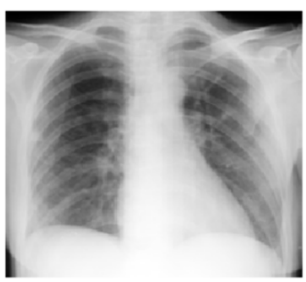

(m)

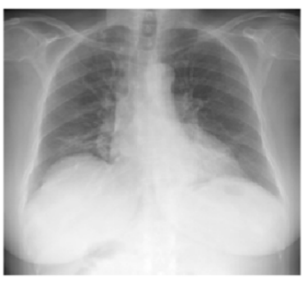

(q)
Ground truth image

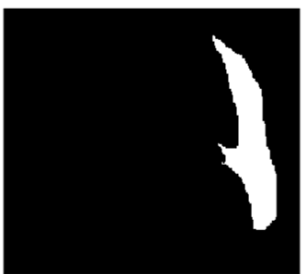

(b)

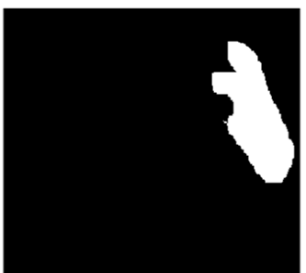

(f)

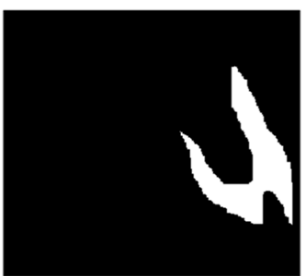

(j)

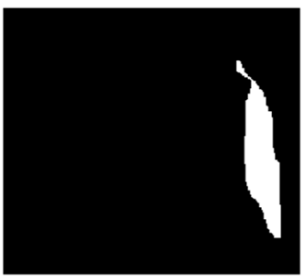

(n)

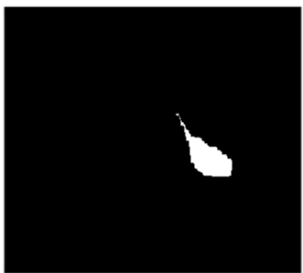

(r)
Affected part tracking
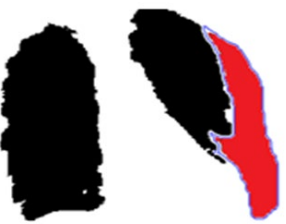

(c)

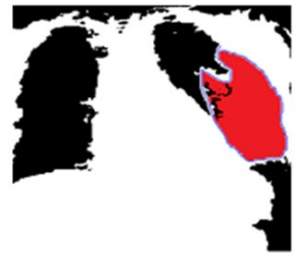

(g)

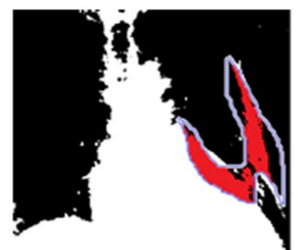

(k)

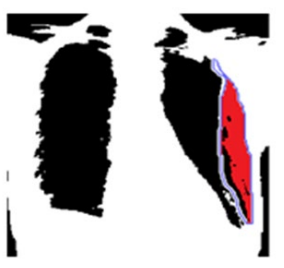

(o)

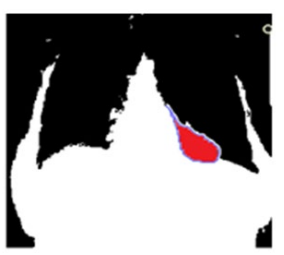

(s)
Segmented image

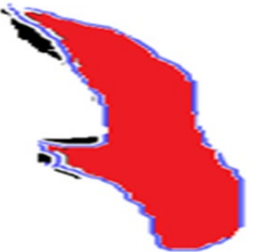

(d)

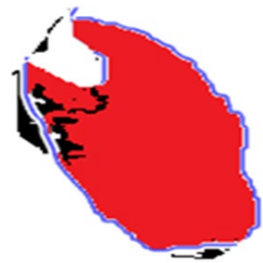

(h)

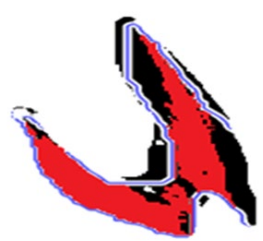

(I)

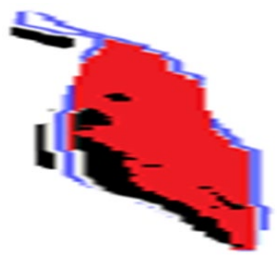

(p)

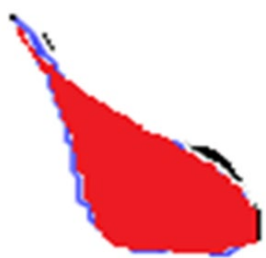

(t)

Fig. 5 Segmentation output by the proposed model a sample-1 input image, b sample -1 ground truth image, c sample-1 affected part tracking image, $\mathbf{d}$ sample-1 segmented image, e sample-2 input image, $\mathbf{f}$ sample-2 ground truth image, $\mathbf{g}$ sample-2 affected part tracking image, $\mathbf{h}$ sample-2 segmented image, $\mathbf{i}$ sample- 3 input image, $\mathbf{j}$ sample-3 ground truth image, $\mathbf{k}$ sample- 3 affected part tracking

image, $\mathbf{l}$ sample-3 segmented image, $\mathbf{m}$ sample-4 input image, $\mathbf{n}$ sample- 4 ground truth image, o sample- 4 affected part tracking image, p sample-4 segmented image, $\mathbf{q}$ sample-5 input image, $\mathbf{r}$ sample-5 ground truth image, $\mathbf{s}$ sample-5 affected part tracking image, $\mathbf{t}$ sample-5

$A_{c c}=\frac{t_{n}^{\prime}+t_{p}^{\prime}}{t_{n^{\prime}}^{\prime}+t_{p^{\prime}}^{\prime}+f_{n^{\prime}}^{\prime}+f_{p^{\prime}}^{\prime}}$,

for patients in a previous stage. Consequently, accuracy classification is mentioned in the following equation: 
Fig. 6 Training accuracy and loss
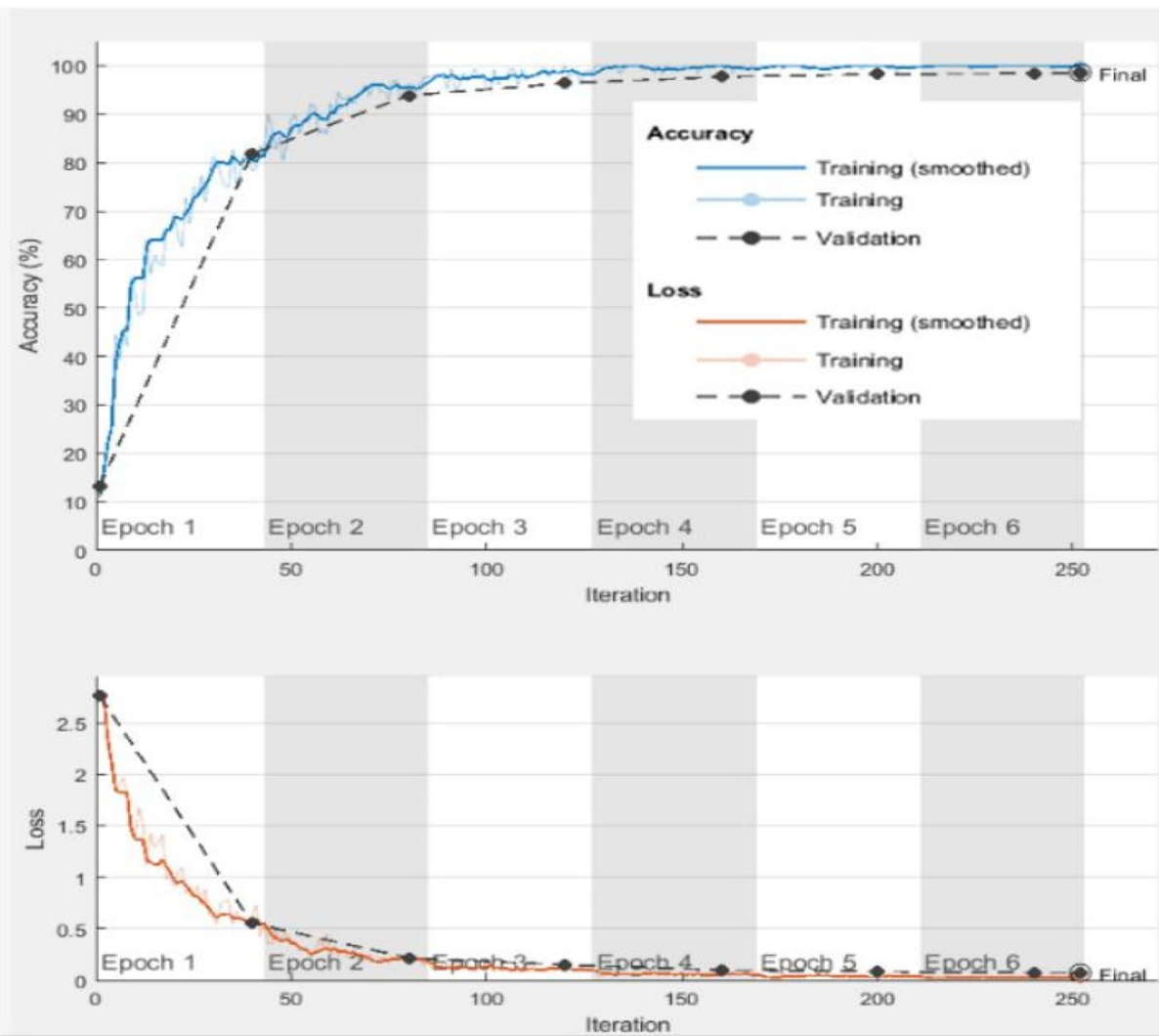

\begin{tabular}{|c|c|c|c|}
\cline { 2 - 4 } \multicolumn{1}{c|}{$\mathrm{N}=1000$} & \multicolumn{2}{c|}{ Predicted } & \multicolumn{1}{c}{} \\
\hline \multirow{3}{*}{ Actual } & $\mathrm{TP}=492$ & $\mathrm{FP}=13$ & 505 \\
\cline { 2 - 4 } & $\mathrm{FN}=5$ & $\mathrm{TP}=490$ & 495 \\
\hline \multirow{2}{*}{} & 497 & 503 & \multicolumn{1}{|c}{} \\
\cline { 2 - 4 } & &
\end{tabular}

Fig. 7 Confusion matrix

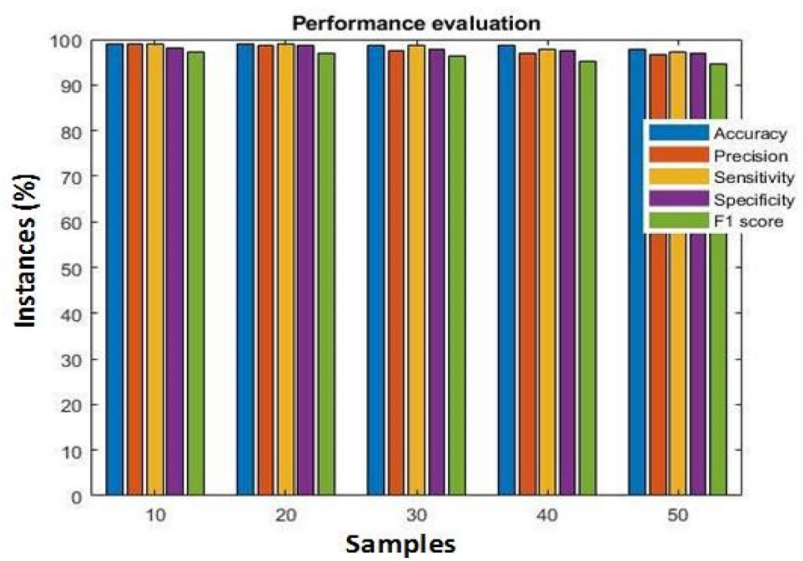

Fig. 8 Overall performance of the proposed method

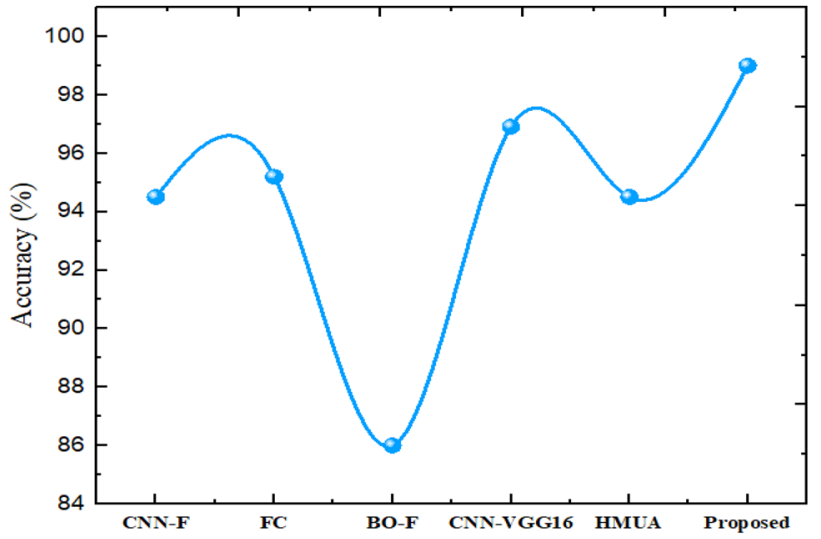

Fig. 9 Overall performance of the proposed method

where $t_{n^{\prime}}^{\prime}, t_{p^{\prime}}^{\prime}$ is determined as true positive and true negative rate consequently, the false-negative rate and the false positive rate is denoted as $f_{n^{\prime}}^{\prime}, f_{p^{\prime}}^{\prime}$.

Comparing other approaches, the proposed model achieved a $99 \%$ higher accuracy than other models, as shown in Fig. 9. 


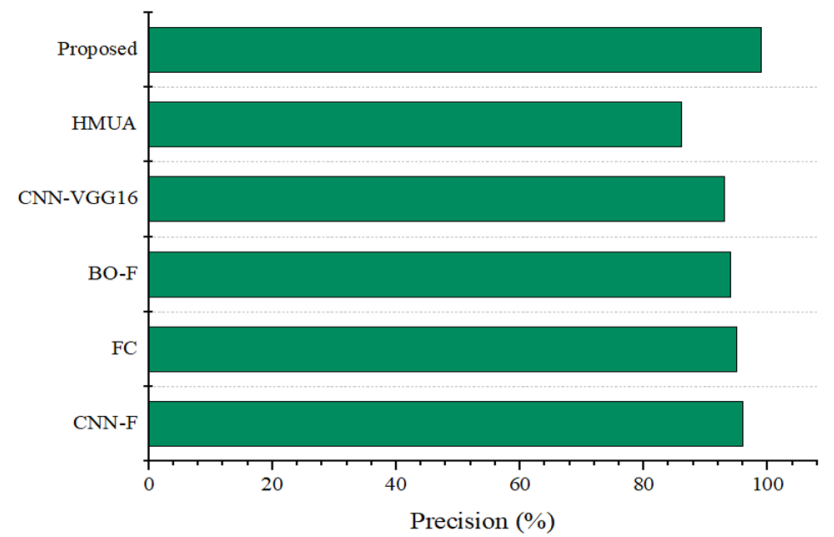

Fig. 10 Comparison performance of precision

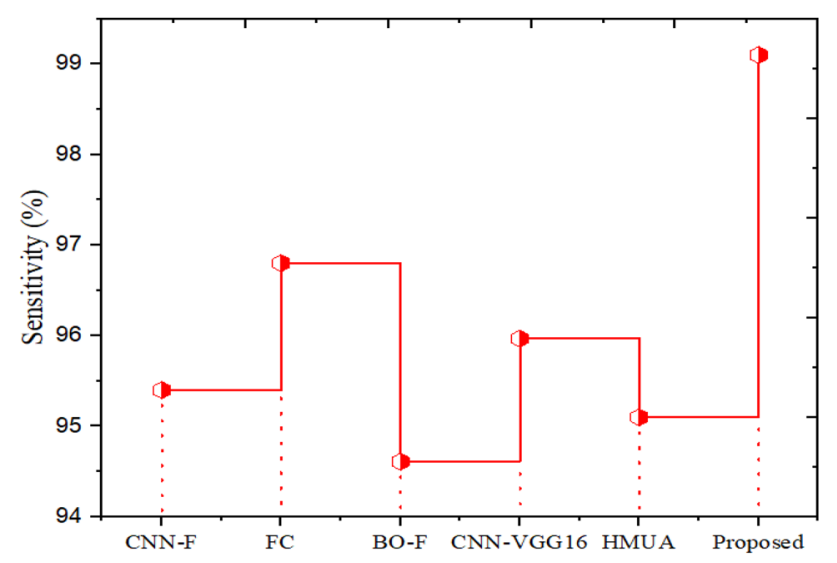

Fig. 11 Comparison performance of sensitivity

\subsubsection{Precision}

Precision is identifying the number of exact positive rates separated by the entire positive rates. Moreover, Precision is the analysis or identification of severe COVID-19 disease, as mentioned in the following equation:

$P=\frac{\left(t_{p^{\prime}}^{\prime}\right)}{\left(t_{p^{\prime}}^{\prime}+f_{n^{\prime}}^{\prime}\right)}$.

The Precision is validated by classifying the detected symptoms by a total number of trained symptoms for COVID-19 disease. Moreover, the developed model achieved a precision rate of $99 \%$ for particular data sets, as shown in Fig. 10.

In the comparison, Fig. 11, the existing models such as CNN-F attained a Precision as 96\% FC pertained Precision as $95 \%$, BOF has gained 94\% Precision rate and HMUA has acquired 86\% Precision and CNN with VGG16 has achieved
93\% Precision. However, the proposed technique has attained 99\% precision, which is quite better than another model.

\subsubsection{Sensitivity}

Sensitivity is described based on the exact disease prediction that is identified by true positive rates. Moreover, sensitivity is proportional to the true positive rate of properly identified severity of COVID-19 disease and inversely proportional to the summation of false and true positive rates, which is mentioned in the following equation:

$S_{n}=\frac{\left(t_{p^{\prime}}^{\prime}\right)}{\left(t_{p^{\prime}}^{\prime}+t_{n^{\prime}}^{\prime}\right)}$.

The proposed strategy has gained a sensitivity rate of 99.1\%, which is shown in Fig. 11. Concurrently, CNN-F attained Sensitivity as $95.4 \% \mathrm{FC}$ pertainedSensitivity as 96.8\%, BOF has gained $94.6 \%$ Sensitivity rate, and HMUA has attained $95.1 \%$ Sensitivity, and CNN with VGG16 has achieved $95.97 \%$ Sensitivity.

\subsubsection{Specificity}

Specificity is the measure of the true negative rate. In addition, specificity is the ratio of wrongly classified severity of COVID-19 disease to the total amount of true negative and false-positive rates. Specificity can be calculated by the following equation:

$S_{p}=\frac{t_{n^{\prime}}^{\prime}}{t_{n^{\prime}}^{\prime}+f_{p^{\prime}}^{\prime}}$.

The proposed VbAFN strategy gained a specificity rate of 98.02\%, and the comparison of statistics is detailed in Fig. 12. Simultaneously, CNN-F attained Specificity as $93.7 \%$ FC pertainedSpecificity as $93.4 \%$, BOF has gained $96 \%$ Specificity,

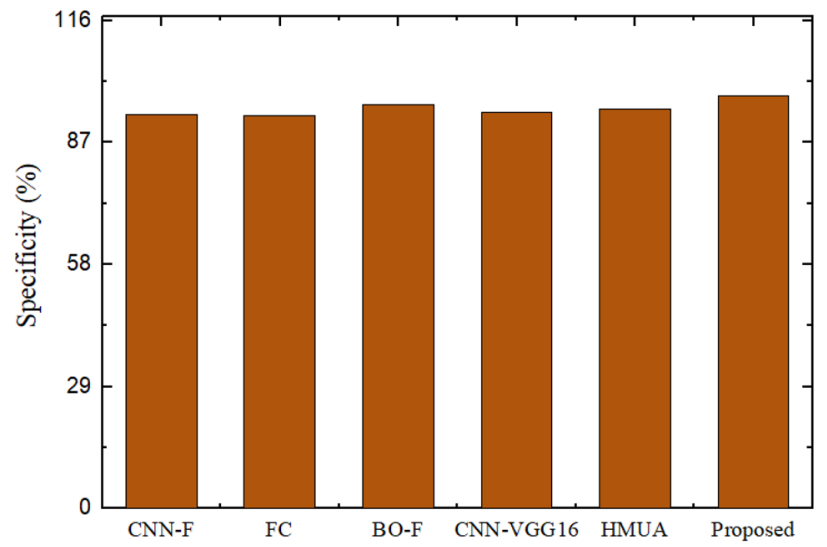

Fig. 12 Comparison performance of specificity 


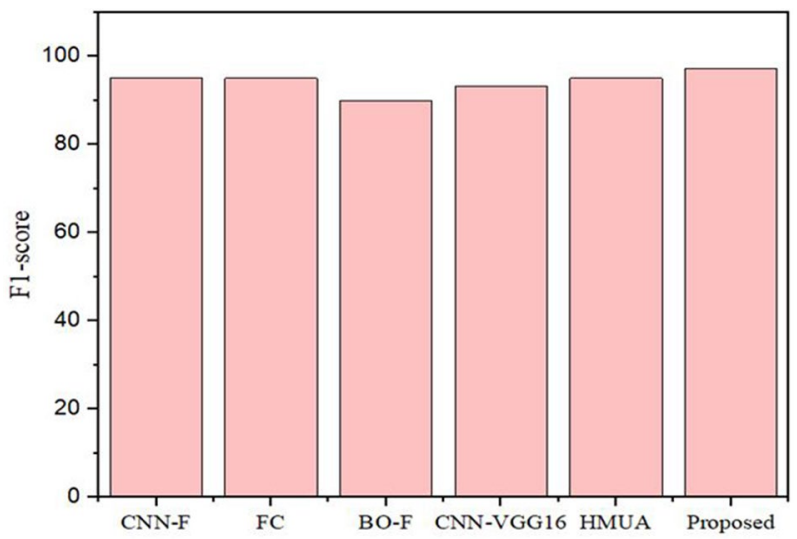

Fig. 13 F1-score comparison

and HMUA has attained 95\% Specificity and CNN with VGG16 has achieved $94 \%$ Specificity.

\subsubsection{F1-score}

F1-score is the important parameter is efficiently tracking the affected region in collected data sets. Moreover, F1-score is directly proportional to the properly classified true positive rate and inversely proportional to the summation of true and false positive values and false negative values, as mentioned in the following equation:

$F 1 \_$Score $=\frac{2 *\left(t_{p^{\prime}}^{\prime}\right)}{2 *\left(t_{p^{\prime}}^{\prime}+f_{p^{\prime}}^{\prime}+f_{n^{\prime}}^{\prime}\right)}$.

The F1-score is compared with various methods such as $\mathrm{CNN}$, ensemble classifier, and fusion schemes which are represented in Fig. 13. The comparison of F1-score in the proposed system is highly improved as $97.23 \%$. Simultaneously, CNN-F attained an F-measure as $95.1 \%$, FC pertained $F$ value as $94.98 \%$, BOF has gained $90 \% F$ value rate, and HMUA has attained $95 \% F$ value and CNN with VGG16 has achieved 93.23\% F-measure.

\subsubsection{Error Rate}

The error rate is evaluated for recognizing the difference between the accurate false positive rates are inversely proportional to the total affected regions is termed s error rate, which is calculated using the following equation:

Error_rate $=\frac{f_{p}^{\prime}+f_{n}^{\prime}}{\text { total }}$.

The proposed VbAFN model gained a lower error rate of 0.014 , while compared with the existing approach, it achieved

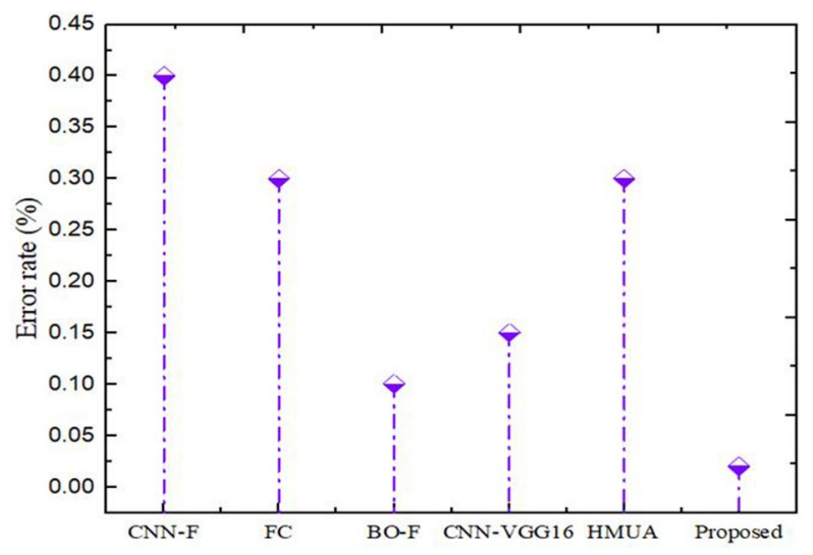

Fig. 14 Comparison performance of error rate

a very less error rate. Simultaneously, the existing method $\mathrm{CNN}-\mathrm{F}$ attained an error rate of $0.4 \%, \mathrm{FC}$ pertained error rate as $0.3 \%, \mathrm{CNN}$ with VGG16 has gained $0.15 \%$ error rate, BO-F has achieved $0.1 \%$ error rate and HMUA has attained $0.3 \%$ error rate. In addition, a comparison of error rates is demonstrated in Fig. 14.

The overall discussed comparison statistics are tabulated in Table 1. The designed model has gained better results for all metrics, which shows the system's reliability. In addition, the designed model has the ability to segment the affected part in the unseen chest X-ray data.

\section{Discussion}

Several conventional techniques are explained and their outcomes are discussed and compared with other models. Hence, this state of the art has helped to identify the future work by identifying the demerits of this current projected work. The demerits and merits of the existing model are described in Table 2.

Accuracy and Precision are high compared to conventional techniques. Therefore, the proposed VbANF model effectively improves the performance. Moreover, the overall performance proposed method is detailed in Table 3. Moreover, the values in Table 3 have been gained by varying the training and testing samples ratio. These training and testing samples have included normal and abnormal chest X-ray images. For the feature extraction and segmentation process, the abnormal images were considered.

The entire comparison demonstrates that the proposed VbANF replica has achieved the finest results through sensitivity, specificity, etc. Thus, the robustness and need of this proposed design in the medical system is proved. 
Table 1 Statistics of comparison

\begin{tabular}{lllllll}
\hline Technique & \multicolumn{2}{l}{ Comparison statistics } & & \\
\cline { 2 - 7 } & F1-score (\%) & Accuracy (\%) & Precision (\%) & Sensitivity (\%) & Specificity (\%) & Error rate (\%) \\
\hline CNN-F & 95.1 & 94.5 & 96 & 95.4 & 93.7 & 0.4 \\
FC & 94.98 & 95.2 & 95 & 96.8 & 93.4 & 0.3 \\
BO-F & 90 & 86 & 94 & 94.61 & 96 & 0.1 \\
CNN with VGG16 & 93.23 & 96.91 & 93 & 95.97 & 94 & 0.15 \\
HMUA & 95 & 94.5 & 86 & 95.1 & 95 & 0.3 \\
Proposed (VbANF) & 97.23 & 99 & 99 & 99.1 & 98.02 & 0.02 \\
\hline
\end{tabular}

Table 2 State-of-the-art comparison

\begin{tabular}{|c|c|c|c|}
\hline Author name & Technique & Advantages & Disadvantages \\
\hline Nour et al. [21] & CNN-F & $\begin{array}{l}\text { Process successfully gives } 98.5 \text { percentage of accuracy } \\
\text { value }\end{array}$ & It has taken more time to run the entire process \\
\hline Zebari et al. [22] & FS & The proposal helps the radiologist & $\begin{array}{l}\text { The determination accuracy depends on the } \\
\text { quality of data in KNN }\end{array}$ \\
\hline Kaur et al. [23] & $\mathrm{BO}-\mathrm{F}$ & $\begin{array}{l}\text { Health care data sets are used to attain correct predic- } \\
\text { tion }\end{array}$ & Overlapping occurs in the target classes \\
\hline Heidari et al. [24] & CNN and VGG16 & Possible to use the large data set & High correlation and high matrix dimensionality \\
\hline Marfak et al. [25] & HMUA & Computation time low & Inaccurate segmentation \\
\hline Proposed & VbANF & $\begin{array}{l}\text { High accuracy and F1-score, High Precision, High } \\
\text { sensitivity, and specificity, lower error rate }\end{array}$ & - \\
\hline
\end{tabular}

Table 3 Outcomes of the proposed method

\begin{tabular}{llllllll}
\hline $\begin{array}{l}\text { No. of trained } \\
\text { samples }\end{array}$ & Test samples & Accuracy (\%) & Precision (\%) & Sensitivity (\%) & Specificity (\%) & F1 score (\%) & Error rate (\%) \\
\hline 7000 & 3000 & 99 & 99 & 99.1 & 98.02 & 97.23 & 0.014 \\
6500 & 3500 & 98.9 & 98.56 & 98.97 & 98.57 & 97 & 0.019 \\
6000 & 4000 & 98.77 & 97.48 & 98.56 & 97.93 & 96.28 & 0.024 \\
5500 & 4500 & 98.58 & 97 & 97.68 & 97.64 & 95.26 & 0.026 \\
5000 & 5000 & 97.83 & 96.76 & 97.37 & 97.06 & 94.56 & 0.028 \\
\hline
\end{tabular}

\section{Conclusion}

In the modern era, image processing is one kind of interesting subject in medical functions. In some cases, the prediction and classification of the disease may be complex. In the modern era, COVID-19 is a severe healthaffected disease, and it's increasing every day. Therefore, this paper proposed a new VbANF technique to estimate the severity of the COVID-19 in an early stage. Primarily, the data was trained to the system, and the training errors were eliminated in the preprocessing layer. Then, the error-cleared data enters the classification layer to tract the affected region and segment it. Finally, the proposed VbANF has earned the highest exactness score of $99 \%$ and the lowest error rate of $0.014 \%$, which is quite better than other models. In addition, comparing the existing works, the proposed design has improved the prediction and segmentation accuracy up to $5 \%$ and reduced the error rate up to $1 \%$. Thus, the proposed system is applicable in medical applications.

Acknowledgements None.

Data Availability Data sharing not applicable to this article as no data sets were generated or analysed during the current study. 


\section{Declarations}

Conflict of Interest The authors declare that they have no potential conflict of interest.

Statement of Human and Animal Rights Ethical Approval: all applicable institutional and/or national guidelines for the care and use of animals were followed.

Informed Consent For this type of study, formal consent is not required.

\section{References}

1. Tandel GS, Balestrieri A, Jujaray T, Khanna NN, Saba L, Suri JS (2020) Multiclass magnetic resonance imaging COVID 19 classification using artificial intelligence paradigm. Comput Biol Med 122:103804. https://doi.org/10.1016/j.compbiomed.2020.103804

2. Ghassemi N, Shoeibi A, Rouhani M (2020) Deep neural network with generative adversarial networks pre-training for COVID 19 classification based on MR images. Biomed Signal Process Control 57:101678. https://doi.org/10.1016/j.bspc.2019.101678

3. Yin B, Wang C, Abza F (2020) New COVID 19 classification method based on an improved version of whale optimization algorithm. Biomed Signal Process Control 56:101728. https://doi.org/ 10.1016/j.bspc.2019.101728

4. Parnian A, Mohammadi A, Plataniotis KN (2020) BayesCap: a bayesian approach to COVID 19 classification using capsule networks. IEEE Signal Process Lett 27:2024-2028. https://doi.org/ 10.1109/LSP.2020.3034858

5. Raja PMS (2020) COVID 19 classification using a hybrid deep autoencoder with Bayesian fuzzy clustering-based segmentation approach. Biocybern Biomed Eng 40(1):440-453. https://doi.org/ 10.1016/j.bbe.2020.01.006

6. Singh R, Goel A, Raghuvanshi DK (2020) Computer-aided diagnostic network for COVID 19 classification employing modulated Gabor filter banks. Vis Comput. https://doi.org/10.1007/ s00371-020-01977-4

7. Kalpana R, Chandrasekar P (2020) An optimized technique for COVID 19 classification and detection with radiation dosage calculation in MR image. Microprocess Microsyst 72:102903. https://doi.org/10.1016/j.micpro.2019.102903

8. Muhammad S et al (2020) COVID 19 detection based on extreme learning. Neural Comput Appln 1-13

9. Toğaçar M, Ergen B, Cömert Z (2020) BrainMRNet: COVID 19 detection using magnetic resonance images with a novel convolutional neural network model. Med Hypotheses 134:109531. https://doi.org/10.1016/j.mehy.2019.109531

10. Chandra SK, Bajpai MK (2020) Fractional mesh-free linear diffusion method for image enhancement and segmentation for automatic tumor classification. Biomed Signal Process Control 58:101841. https://doi.org/10.1016/j.bspc.2019.101841

11. Khan H, Shah PM, Shah MA, Islam S, Rodrigues JJPC (2020) Cascading handcrafted features and Convolutional Neural Network for IoT-enabled COVID 19 segmentation. Comput Commun 153:196-207. https://doi.org/10.1016/j.comcom.2020.01.013

12. Kaur G, Oberoi A (2020) Novel approach for COVID 19 detection based on Naïve Bayes classification. Data Management, Analytics and Innovation, Springer, Singapore, pp 451-462. https://doi.org/ 10.1007/978-981-32-9949-8 31

13. Polepak S, Rao CS, Mohan MC (2020) IDSS-based Two stage classification of COVID 19 using SVM. Health Technol 10(1):249-325. https://doi.org/10.1007/s12553-018-00290-4

14. Sahoo L, Sarangi L, Dash BR, Palo HK (2020) Detection and classification of COVID 19 using magnetic resonance images. Advances in electrical control and signal systems, Springer, Singapore, pp 429-441. https://doi.org/10.1007/978981-15-5262-5_31

15. Toğaçar M, Cömert Z, Ergen B (2020) Classification of brain MRI using hyper column technique with convolutional neural network and feature selection method. Expert Syst Appl 149:113274. https://doi.org/10.1016/j.eswa.2020.113274

16. Ghahfarrokhi SS, Khodadadi H (2020) Human COVID 19 diagnosis using the combination of the complexity measures and texture features through magnetic resonance image. Biomed Signal Process Control 61:102025. https://doi.org/10.1016/j.bspc.2020. 102025

17. Khan SR, Sikandar M, Almogren A, Din IU, Guerrieri A, Fortino G (2020) IoMT-based computational approach for detecting COVID 19. Future Gener Comput Syst 109:360-367. https://doi. org/10.1016/j.future.2020.03.054

18. Jia Z, Chen D (2020) COVID 19 Identification and Classification of MRI images using deep learning techniques. IEEE Access. https://doi.org/10.1109/ACCESS.2020.3016319

19. Hashemzehi R, Mahdavi SJS, Kheirabadi M, Kamel SR (2020) Detection of COVID 19s from MRI images base on deep learning using hybrid model CNN and NADE. Biocybern Biomed Eng 40(3):1225-1232. https://doi.org/10.1016/j.bbe.2020.06.001

20. Meenakshi A, Revathy S (2020) An Efficient Model for Predicting COVID 19 using Deep Learning Techniques. 2020 5th International Conference on Communication and Electronics Systems (ICCES), IEEE. https://doi.org/10.1109/ICCES48766.2020.91380 29

21. Nour M, Cömert Z, Polat K (2020) A novel medical diagnosis model for COVID 19 infection detection based on deep features and Bayesian optimization. Appl Soft Comput 97:106580. https:// doi.org/10.1016/j.asoc.2020.106580

22. Zebari DA, Abdulazeez AM, Zeebaree DQ, Salih MS (2020) A Fusion Scheme of Texture Features for COVID 19 Detection of CT Scan Images. 2020 International Conference on Advanced Science and Engineering (ICOASE), IEEE. https://doi.org/10.1109/ ICOASE51841.2020.9436538

23. Kaur T, Gandhi TK, Panigrahi BK (2021) Automated diagnosis of COVID 19 using deep features and parameter free BAT optimization. IEEE J Transl Eng Health Med 9:1-9. https://doi.org/ 10.1109/JTEHM.2021.3077142

24. Heidari M, Mirniaharikandehei S, Khuzani AZ, Danala G, Qiu Y, Zheng B (2020) Improving the performance of CNN to predict the likelihood of COVID 19 using chest X-ray images with preprocessing algorithms. Int J Med Inform 144:104284. https://doi.org/ 10.1016/j.ijmedinf.2020.104284

25. Marfak A, Achak D, Azizi A, Nejjari C, Aboudi K, Saad E, Hilali A, Youlyouz-Marfak I (2020) The hidden Markov chain modelling of the COVID 19 spreading using Moroccan dataset. Data Brief 32:106067. https://doi.org/10.1016/j.dib.2020.106067

26. Shang Y, Liu T, Wei Y et al (2020) Scoring systems for predicting mortality for severe patients with COVID-19. EClin Med 24:100426. https://doi.org/10.1016/j.eclinm.2020.100426

27. Cheema TN, Raja MAZ, Ahmad I et al (2020) Intelligent computing with Levenberg-Marquardt artificial neural networks for nonlinear system of COVID-19 epidemic model for future generation 
disease control. Eur Phys J Plus 135(11):1-35. https://doi.org/10. 1140/epjp/s13360-020-00910-x

28. Shoaib M, Salahudin H, Hammad M et al (2021) Performance evaluation of soft computing approaches for forecasting COVID19 pandemic cases. SN Comput Sci 2(5):1-13. https://doi.org/10. 1007/s42979-021-00764-9

29. Zhang L, Yan X, Fan Q et al (2020) D-dimer levels on admission to predict in-hospital mortality in patients with Covid-19. J Thromb Haemost 18(6):1324-1329. https://doi.org/10.1111/jth. 14859
30. Shanbhag V, Arjun NR, Chaudhuri S et al (2021) Utility of age-adjusted charlson comorbidity index as a predictor of need for invasive mechanical ventilation, length of hospital stay, and survival in COVID-19 patients. Indian J Crit Care Med 25(9):987-991

31. Kaggle X-ray data assessed at: https://www.kaggle.com/ praveengovi/coron ahack-chest- $x$ rayd ataset? select $=$ Coron ahack-Chest-XRay- Dataset

\section{Authors and Affiliations}

\section{S. Roselin Mary ${ }^{1}$ - Vinit Kumar ${ }^{2}$ K. J. Prasanna Venkatesan ${ }^{3} \cdot$ R. Satish Kumar ${ }^{4}$. Naga Padmaja Jagini ${ }^{5}$. Amedapu Srinivas ${ }^{6}$}

S. Roselin Mary jesuroselin@gmail.com

Vinit Kumar dr.vinitkumar76@gmail.com

K. J. Prasanna Venkatesan prasanna@nec.edu.in

\section{R. Satish Kumar} harsiga@gmail.com

Naga Padmaja Jagini srija26@gmail.com

1 Department of Computer Science and Engineering, Anand Institute of Higher Technology, Chennai, Tamilnadu 603103, India
2 Department of Computer Science and Engineering, Galgotias College of Engineering and Technology, Greater Noida 201310, India

3 Department of Electronics and Communication Engineering, National Engineering College, Kovilpatti, Tamilnadu 628503, India

4 Department of Medical Electronics, Sengunthar College of Engineering, Tiruchengode, Tamilnadu 637205, India

5 Department of Computer Science and Engineering, Vardhaman College of Engineering, Hyderabad, Telangana 501218, India

6 National Institute of Technology, Trichy, Tamilnadu 620015, India 